\title{
Prospects for the Rapid Detection of Mealiness in Apples by Nondestructive NMR Relaxometry
}

\author{
P. Barreiro', A. Moya', E. Correa ${ }^{1}$, M. Ruiz-Altisent', \\ M. Fernández-Valle ${ }^{2}$, A. Peirs ${ }^{3}$, K. M. Wright ${ }^{4}$, and B. P. Hills ${ }^{4}$ \\ ' Deparnamento de Ingeniería Rural, Universidad Politécnica de Madrid, Madrid, Spain \\ ${ }^{2}$ Centro de Resonancia Magnética Nuclear, Universidad Complutense de Madrid, Madrid, Spain \\ ${ }^{3}$ Flanders Center/Laboratory of Postharvest Technology, Katholieke Universiteit Leuven, \\ Leuven, Belgium \\ ${ }^{4}$ Institute of Food Research, Norwich Research Park, Norwich, UK
}

\begin{abstract}
The potential of nuclear magnetic resonance (NMR) relaxometry for quantitative evaluation of apple mealiness has been investigated. The degree of "mealiness" was defined by several mechanical techniques, including penetration, compression and shear rupture as well as by the BRIX (soluble solids) and juiciness levels. These data were cortelated with both magnetic resonance imaging (MRI) and NMR water proton transverse relaxation time measurements on a fruit-by-fruit basis. It was found that increasing mealiness caused a systematic increase in the transverse relaxation rate. The potential for rapid, on-line NMR/MRI detection of appie mealiness is discussed.
\end{abstract}

\section{Introduction}

The idea of developing nuclear magnetic resonance (NMR) or magnetic resonance imaging (MRI) as sensors of internal defects in fruit is an attractive one but first requires that the internal quality factor of interest, such as ripeness, mealiness or juiciness is strongly correlated with an NMR measureable parameter, such as a relaxation time or spin density. In this paper we focus on the internal quality of apples and explore whether there is a significant correlation between the water proton transverse relaxation behavior and quality factors such as mealiness. Preliminary reports by Barreiro et al. in 1999 and $2000[1,2]$ succeeded in establishing a correlation between mealiness and changes in the spatially resolved $T_{2}$ maps in apples derived from sequences of 20 spin-echo images. Unfortunately, acquiring 20 images of each apple is quite impractical for the purpose of sorting apples on the basis of their mealiness so, in this paper, we explore whether nonspatially resolved relaxation measurements can be correlated with spatially resolved relaxation time distributions and mealiness. This idea gains additional 
credence from the earlier work by Hills and Remigereau [3], who showed that the distribution of water proton transverse relaxation times for parenchyma apple tissue was sensitive to the subcellular distribution of water. Nonspatially resolved relaxation time distributions can be acquired much faster than $T_{2}$ maps and can also be acquired at low field. Significant correlations between mealiness and transverse relaxation times would therefore open up the possibility of rapid, low-cost on-line applications. In this paper we therefore investigate whether such correlations exist and also how they compare with the MRI $T_{2}$ histograms. When dealing with fruit quality it is necessary to perform statistical correlations over large numbers of samples. For this reason the NMR measurements were shared between three centres, namely, the Flanders Laboratory of Postharvest Technology (VCBT), the Universidad Politécnica de Madrid (UPM), the Universidad Complutense de Madrid (UCM) and the Institute of Food Research in Norwich (IFR), who collaborated under an EC-funded project (FAIR CT 95-0302) [4-6].

Although the present study is confined to apples, similar NMR methods could be applied to other types of fruit, for example, the detection of woolliness (mealiness) in peaches. It would also be interesting to compare NMR methods and sensitivity with other spectroscopic methods such as near-infrared (NIR) spectroscopy. Indeed Ortiz et al. [7] has already investigated the combination of NIR and nondestructive impacts for the identification of woolliness in peaches, but NMR methods remain to be applied and compared with the NIR data.

\section{Experimental Methods}

\section{I Magnetic Resonance Imaging (UCM-UPM)}

MRI experiments were performed at UCM (Madrid) on a Bruker Biospec 47/40 (Ettlingen, Germany) spectrometer equipped with actively shielded imaging gradients capable of reaching $370 \mathrm{mT} / \mathrm{m}$ along all axes. The bore size of the magnet was $40 \mathrm{~cm}$, but with the gradient stack in place the internal bore diameter was reduced to $12 \mathrm{~cm}$. A home-built high-pass birdcage coil $(N=8)$ with an inner diameter of $10.4 \mathrm{~cm}$ and a length of $14.0 \mathrm{~cm}$ was constructed to adapt the coil to the maximum size of our apples.

Apples were imaged as 128 by 128 matrices with the standard two-dimensional (2-D) spin echo sequence. 1 slice and 20 echoes were collected with an echo time, TE, of $9.5 \mathrm{~ms}$ and a fixed repetition time of $3000 \mathrm{~ms}$. A $5 \mathrm{~mm}$ slice thickness and a $10 \mathrm{~cm}$ field of view (FOV) were used. Pulse gains, attenuator and shimming settings were previously adjusted for a $6 \mathrm{~cm}$ sphere filled with doped water (copper sulphate, $1 \% \mathrm{w} / \mathrm{w}$ ) and were held constant along the total experiment, in which several apples were imaged under these conditions. The field homogeneity was tested as the lack of change in the images of the spherical phantom before and after the apples recording. For comparison, all images were reconstructed with an identical scale factor. The magnitude of these multiecho images was fitted on a pixel-by-pixel basis with a two-parameter $\left(T_{2}\right.$ and the sig- 
nal intensity at equilibrium) monoexponential function without baseline, with the standard Bruker imaging Fit Package, which uses the Levenberg-Marquart criterion for chi-square minimization operating on a Silicon Graphics Indy computer. The software package allows adjustment of the threshold so as to remove those pixels with low signal-to-noise $(\mathrm{S} / \mathrm{N})$ ratio. In this way $T_{2}$ maps of all samples were obtained after removal of all pixels under the $15 \%$ of the maximum signal intensity, which successfully removed background pixels. Histograms were obtained by removing those pixels with $T_{2}$ values (digitized at four bits) of zero corresponding to the core and background areas of the apple images.

Following previous research work [2] these data were used to calculate the following subsidiary parameters: The average, standard deviation, maximum and minimum $T_{2}$ values were calculated from the global $T_{2}$ maps (disregarding those pixels corresponding to the background or the core). The $T_{2}$ histograms were used to derive the lower quartile ( $T_{2}$ lower limit containing $25 \%$ of pixels of the histogram), upper quartile ( $T_{2}$ upper limit containing $25 \%$ of pixels of the histogram), $T_{2}$ mode (most frequent values), mode height (number of pixels with $T_{2}$ value equal to the mode), interquartile range (difference between upper and lower quartiles), first shape coefficient (mode height/interquartile range), and second shape coefficient ( 0.5 times the number of pixels of the histogram/interquartile range).

\subsection{NMR Relaxation (IFR)}

NMR proton transverse relaxation measurements were carried out on a Bruker MSL100 spectrometer operating at $100 \mathrm{MHz}$ with a thermostated, high-power probe with a $90^{\circ}$ pulse length of $2 \mu s$ and the Carr-Purcell-Meiboom-Gill (CPMG) pulse sequence with a $90-180^{\circ}$ pulse spacing of $200 \mu \mathrm{s}$. Data were averaged over eight acquisitions at a recycle delay of $10 \mathrm{~s}$ to avoid saturation. This protocol has been extensively investigated on apples in earlier studies [6]. Each measurement was destructive because the probe only permitted $5 \mathrm{~mm}$ sample diameters. Two repetitions per fruit were performed on the blushed and green area of the fruits in the 1999 batch and 3 repetitions in the 2000 batch.

The CPMG echo-delay envelopes were analyzed as a continuous distribution of exponential relaxation times by the method described by Provencher and Dovi [8] and Provencher [9]. Thus the time domain signal is transformed into a $r_{2}$ domain signal. This continuous distribution of exponential relaxation times gives information comparable to that of $T_{2}$ histograms from MRI, though it is important to note that the CPMG echo decays have 1024 echoes with a short pulse spacing, while the MRI echo decays have 20 echoes and a longer pulse spacing.

\subsection{NMR Relaxation (UCM-UPM)}

The proton transverse relaxation behavior of the second apple batch was also analyzed at UCM with a Bruker Biospec $47 / 40$ operating at $200 \mathrm{MHz}$ with a 
$90^{\circ}$ pulse length of $130 \mu$ s on whole fruits. The CPMG pulse sequence was again used with a $90-180^{\circ}$ pulse spacing of $200 \mu$ s (1024 points per curve, total duration of $3 \mathrm{~s}$ ). No data averaging was performed. Time delay between measurements was $10 \mathrm{~s}$ to avoid saturation. Note that the pulse spacing is equal to that of the IFR equipment, while the pulse length is far longer (130 $\mu \mathrm{s}$ compared to $2 \mu \mathrm{s})$. The spin-locking time is therefore a significant fraction of the acquisition time and the measurement assumes more the characteristics of a $T_{\text {ltho }}$ (the rotating frame relaxation time) measurement. The echo decay envelopes were analyzed as a continuous distribution of exponential relaxation times [1, 6] at IFR. Because measurements were taken on the whole fruit three repetitions were performed per fruit and the $6 \mathrm{~cm}$ sphere filled with doped water (copper sulphate, $1 \% \mathrm{w} / \mathrm{w}$ ) was used as a control with three repetitions both at the beginning and the end of each of the three test sessions.

\subsection{Fruit}

Cox's Orange Pippin apples were submitted by the Flanders Center/Laboratory of Postharvest Technology (VCBT) to an accelerated test to enhance mealiness [4] at three potential mealiness levels (level 0 , nonmealy apples; level 1 , medium stag; level 2, mealy apples) in two consecutive seasons (1999 and 2000). In 2000 , a fourth batch of fruit of unknown mealiness stage was gathered from three different commercial stores, in order to compare the textural properties with those of the apples submitted to the accelerated test. These samples were generated with the support of the European Concerted Action ASTEQ.

Altogether 120 apples have been analyzed in this study over two growing seasons. The first set of measurements was undertaken at the IFR in 1999 and the second batch was analyzed at the VCBT in 2000 . In the 2000 batch, duplicate samples were transported to IFR and UCM under controlled conditions because NMR equipment was only located at these sites. Table 1 shows a summary of the experimental design in both seasons.

\subsection{Mechanical and Chemical Tests (UPM-VCBT)}

To discover whether the NMR measurements correlate with apple quality, it is necessary to use independent non-NMR instrument methods to quantify the quality factors of interest. The following non-NMR techniques were used.

The Magness-Taylor penetration test was carried out with a Texture Analyzer XT2 (Norwich experiment) and with an LRX testing machine (Leuven experiment) on entire fruits. The Magness-Taylor flesh penetration test was performed with an $8 \mathrm{~mm}$ diameter rod that penetrated the fruit over a maximum of $8 \mathrm{~mm}$ with a speed of $20 \mathrm{~mm} / \mathrm{min}$. The maximum penetration force was registered and was used as Magness-Taylor firmness. One repetition per fruit was performed.

The confined compression test was carried out with the same instrument as the Magness-Taylor test except that cylindrical cores of apple tissue $(1.7 \mathrm{~cm}$ height and 
Table 1. Summary of the experimental design performed in 1999 and 2000 season on Cox's Orange Pippin apples.

\begin{tabular}{|c|c|}
\hline Norwich experiment & Leuven experiment \\
\hline $\begin{array}{l}\text { Factorial experimental design: } \\
\text { mealiness stage ( } 3 \text { levels) } \\
\text { sample size: } 8 \text { fruits/sample } \\
\text { total number of fruits: } 24 \\
\text { NMR relaxometry carried out } \\
\text { on same } 24 \text { fruits }\end{array}$ & $\begin{array}{l}\text { Factorial experimental design: } \\
\text { mealiness stage ( } 3 \text { levels }+1 \text { unknown) } \\
\text { General experiment: } \\
\text { sample size: } 20 \text { fruits/sample } \\
\text { sample codes for level } i: c i-1 \text { to ci-20, } \\
j=0,1,2 \text {, unknown } \\
\text { total number of fruits: } 80 \\
\text { NMR experiment: } \\
\text { duplicate sample for IFR: } 12 \text { fruits (4 per level) } \\
\text { sample codes for level } i \text { c ci-21 to ci-24, } \\
i=0,1,2 \text {, unknown } \\
\text { duplicate sample for UPM: } 16 \text { fruits } \\
\text { (4 per levej + } 4 \text { of unknown level) } \\
\text { sample codes for level } i: \text { ci-24 to ci- } 28 \text {, } \\
j=0,1,2 \text {, unknown }\end{array}$ \\
\hline
\end{tabular}

$1.7 \mathrm{~cm}$ diameter) were used. These cores were placed inside a cylindrical holder of the same height and diameter and compressed with a metal rod with a diameter of $1.53 \mathrm{~cm}$ at a speed of $20 \mathrm{~mm} / \mathrm{min}$ to give a maximum deformation of $2.5 \mathrm{~mm}$. After reaching the maximum deformation, the rod returned at the same speed in the opposite direction. 1 repetition per fruit was performed for Norwich and Leuven general experiments, while 3 repetitions per fruit were performed in the NMR experiment held at UCM-UPM. The following parameters were registered: forceto-deformation ratio $(\mathrm{N} / \mathrm{mm})$ within the elastic regime which was denoted the "compression hardness", and the juice area $\left(\mathrm{mm}^{2}\right)$ of the spot accumulated in a filter paper placed undemeath the probe during the test (denoted the "compression juiciness"). The combination of both measurements was used to compute a texture degradation score $(0-11)$ of which mealiness covers the last stages $(6-11)$ [5].

For the shear rupture test cylindrical segments $(1.4 \mathrm{~cm}$ diameter and $2 \mathrm{~cm}$ height) of fruit flesh were used to test the crispiness of the fruits. The segments were sheared laterally with a velocity of $20 \mathrm{~mm} / \mathrm{min}$ until they ruptured. The maximum force at shear rupture point was registered as a value for shear crispiness $(\mathrm{N})$. Because this is a destructive test, only one repetition could be carried out on each fruit.

For the Brix value ( $\left.{ }^{\circ} \mathrm{Brix}\right)$ the soluble-solid content was measured with a digital refractometer (PR-101 ATAGO) as a value for the sugar content.

\subsection{Metrology}

The standard deviation (STD) of measurements with respect to the sample unit was used as an indicator of repeatability. The evaluation of accuracy implies the use of reproducible reference materials, with known true values for the parameter under evaluation. For MRI and NMR relaxation a sphere filled with copper 
sulphate $(1 \% \mathrm{w} / \mathrm{w})$ was used. The signal-to-noise $(\mathrm{S} / \mathrm{N})$ ratio was used within the sensor technology ambit as an indicator of the quality of measurements. In our study, the standard error $(S E=S T D \sqrt{n})$ of a parameter for a sample unit was taken as an estimator for the noise (nonaddressed variation), while its average value refers to the signal level. Relating a reference measurement to a new developing technique results in a high $\mathrm{S} / \mathrm{N}$ ratio, which may be insufficient. Thus, $12+0.2{ }^{\circ} \mathrm{Brix}$ has an acceptable $\mathrm{S} / \mathrm{N}$ value $(60)$, but we will probably fail in correlating soluble solids from apples varying from 11 to $12{ }^{\circ} \mathrm{Brix}$ to a new developing technique. This is due to the fact that the range of variation or measured range ( $M R$, maximum - minimum value for all analyzed sample units) for the reference measurements dramatically affects the results of comparison. Therefore there is need for defining new metrological features. The $S E / M R$ ratio was proposed to check the feasibility of inter-technique comparison.

\section{Results}

\subsection{Results of NMR Relaxometry at IFR}

The proton transverse relaxation time spectrum obtained by deconvolution of the 1024 CPMG echoes showed a maximum of three relaxation time peaks (Fig. 1). These peaks have been assigned in ref. 3 to water in the vacuole, cell wall and cytoplasm, though there is some averaging of the water signal due to water diffusion between these subcellular compartments. The longest relaxation time peak is
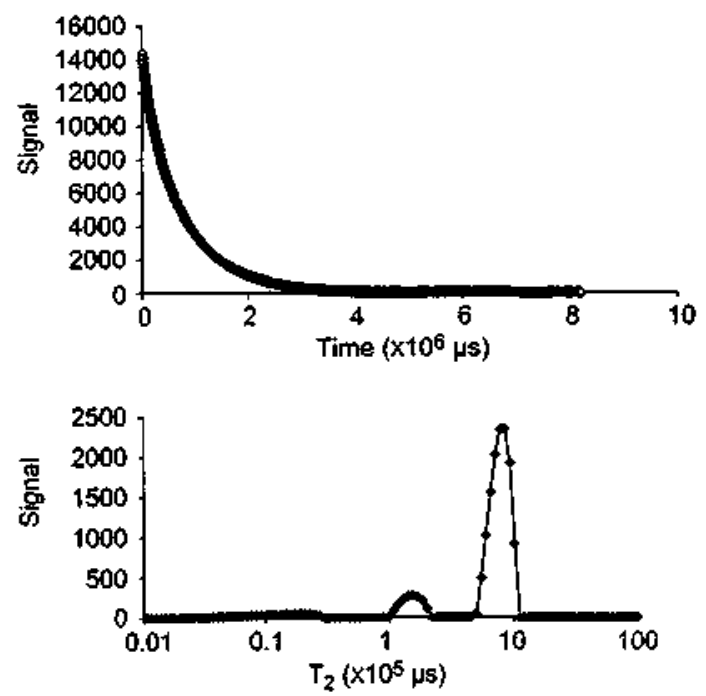

Fig. 1. Example of relaxation curve on apple tissue (top) and result of continuous distribution fitting (bottom). The distance between consecutive points for the continuous distribution fit refers to $T_{2}$ resolution of the results. In this case two peaks are clearly visualized corresponding to different subcellular compartments [3]. 


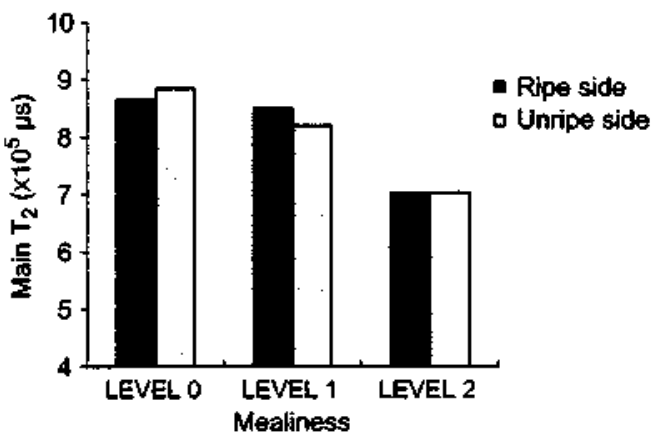

Fig. 2. Main $T_{2}$ peak extracted from NMR relaxation curves on apple tissue. Average per batch is presented ( 8 fruits/batch); $S E= \pm 13433 \mu \mathrm{s}$. Significant differences are found for mealiness level but not for fnuit side. 1999 Norwich general experiment.

dominant and this corresponds mainly to vacuolar water and it can be seen in Fig. 2 that this main relaxation time shows a significant decrease for increasing mealiness level (Fisher value $F=83.01$, probability $p<0.01$ ) in the 1999 batch of samples analyzed at Norwich. Each of these NMR relaxation time measurements were repeated on the blushed (red) and green face of the apple, but the face of the apple made no significant difference. A fruit-by-fruit comparison of the textural references versus the main $T_{2}$ peak of the deconvoluted NMR relaxation curve of the 1999 Norwich batch was attempted without success (linear regression coefficient $r<0.45$ with regard to Magness-Taylor firmness, to hardness, to crispness and to juiciness). This is understandable because of the low SE/MR ratio of the reference measurements (between 0.11 and 0.16 for hardness and juiciness respectively, see Table 2) but not because of a poor performance of the NMR relaxation parameter ( $S E / M R=0.06$ for main $T_{2}$ peak). It was concluded that there was a need for either a broader measured range or a lower standard error in all refer-

Table 2. Metrological features per fruit on some reference measurements.

\begin{tabular}{lccc}
\hline $\begin{array}{l}\text { Feature and } \\
\text { statistic parameter }\end{array}$ & $\begin{array}{c}\text { Norwich experiment } \\
\text { (February 99) }\end{array}$ & $\begin{array}{c}\text { Leuven experiment (January 2000) } \\
\text { General experiment }\end{array}$ \\
\hline NMR experiment at UPM
\end{tabular}

* These values has been derived as $S E \sqrt{3}$ from the experiments held at UPM, as only there three replicates of the measurements were performed per fruit. 


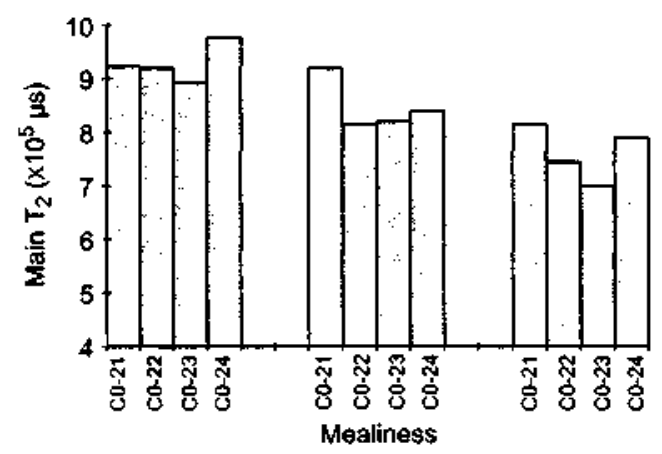

Fig. 3. Main $T_{2}$ peak extracted from NMR relaxation curves. Averages of three replicates per fruit are presented; $S E= \pm 12483 \mu$ s. As for Fig. 2, main $T_{2}$ peak tends to decrease for increasing mealiness. NMR experiment held at IFR is a reduced sample from General experiment 2000.

ence and devoted techniques. The three repetitions used in the 2000 batch experiment led to an improved $S E / M R$ ratio of 0.11 on a per fruit basis. A similar trend was found (Fig. 3), namely, a systematic reduction in the main relaxation time with increasing mealiness.

\subsection{Results of the MRI and NMR Relaxometry at UCM}

The spectrometer performance was first checked with the copper sulphate phantom. The average $T_{2}$ for the copper sulphate solution sphere was $67.9 \mathrm{~ms}$. High spatial homogeneity $( \pm 205 \mu \mathrm{s})$ as well as temporal stability $( \pm 820 \mu$ s within a day and $\pm 1090 \mu$ s between days) was found.

Table 3 shows some metrological features of the NMR relaxometry performed at UCM. Of the three test sessions, two showed very good performance and high reproducibility of the main $T_{2}$ peak $(68.3 \mathrm{~ms})$, while the second test session

Table 3. Accuracy and repeatability test carried out at UPM on the NMR relaxation curves by means of a copper sulphate phantom.

\begin{tabular}{lcc}
\hline Test session nr. and parameter & Beginning of test session & End of test session \\
\hline First & & \\
$\quad$ main $T_{2}$ peak $(\mu \mathrm{s})$ & $68335 \pm 0$ & $68335 \pm 0$ \\
second $T_{2}$ peak $(\mu \mathrm{s})$ & $2287 \pm 1981$ & $3133 \pm 0$ \\
Second & $97760 \pm 40284$ & $43430 \pm 0$ \\
main $T_{2}$ peak $(\mu \mathrm{s})$ & $6284 \pm 324$ & $4645 \pm 246$ \\
second $T_{2}$ peak $(\mu \mathrm{s})$ & $68335 \pm 0$ & $68335 \pm 0$ \\
Third & $9056 \pm 4436$ & $3133 \pm 0$ \\
main $T_{2}$ peak $(\mu \mathrm{s})$ & & \\
second $T_{2}$ peak $(\mu \mathrm{s})$ & &
\end{tabular}


showed abnormal variability. The relaxation data of the second session were therefore discarded in subsequent correlations. The results for the second $T_{\text {z }}$ peak showed less reproducibility than that of main one so they were also disregarded in all subsequent correlations. These results are not surprising given the longer pulse length and the increased contribution of the rotating frame relaxation in this measurement. Note, however, that the average main $T_{2}$ peak derived from deconvoluted NMIR relaxometry curves $(68.3 \mathrm{~ms})$ was very neat to that extracted from $T_{2}$ fitting on MIRI images $(68.0 \mathrm{~ms})$.

Figure 4 shows examples of $T_{2}$ maps for apples with a varying level of mealiness; as before, $T_{2}$ maps were fitted from 20 successive echoes and the mealiness stage was determined by the non-NiMR instrumental references. As with the

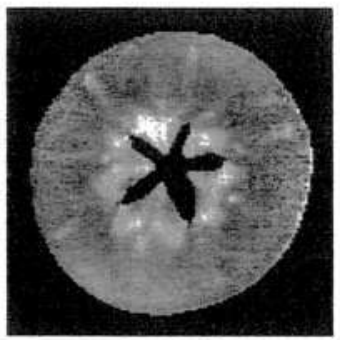

Cox 0-28

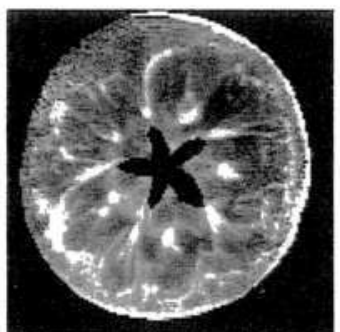

Cox 1-25

e

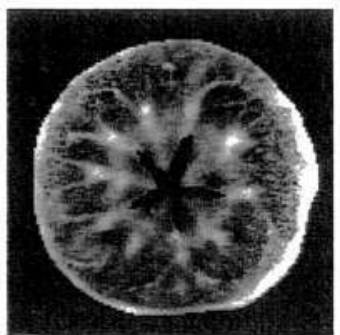

Cox 2-26 b

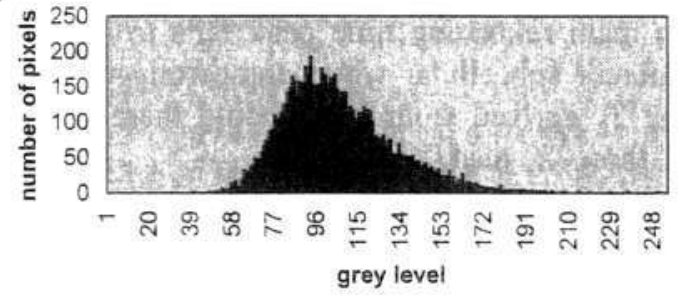

d

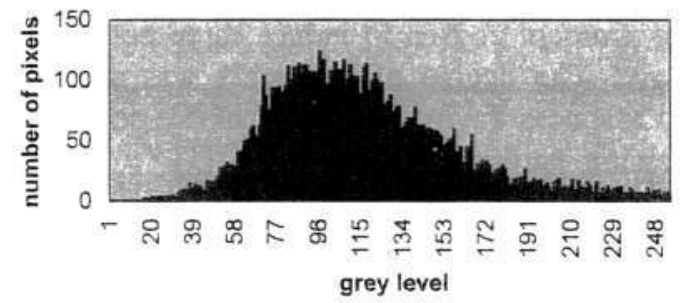

f

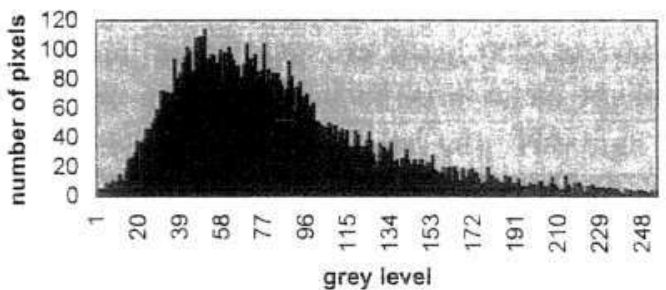

Fig. 4. a Image of Cox apple labelled $0-28$ with a mealiness score of $1.3 ; \mathbf{b}$ bustogram from the $T_{2}$ map of the apple in a. c Image of Cox apple labelled $1-25$ with a mealiness score of 7.9 ; d histogram from the $T_{2}$ map of the apple in c. e Image of Cox apple labelled $2-26$ with a mealiness score of 10.7 ; f histogram. from the $T_{2}$ map of the apple in $\mathbf{e}$. 
nonspatially resolved relaxation data, it was found that the $T_{2}$ decreased with increasing mealiness and that at a certain point a broad change in the histogram shape was observed and corresponds to a significant increase in the standard deviation of the $T_{2}$ values within the apple. These MRl features therefore agreed with expectations on the basis of previous research work $[1,2]$. It is interesting to note that the $T_{2}$ map of apple $\mathrm{C} 0.25$ also feanures a tailed $T_{2}$ histogram and this apple also showed an abnornal variability in texture reference values.

The analysis of the main $T_{2}$ peak from continuous distribution fit of relaxation curves from UCM data on whole fruit reveals similar features as those extracted at IFR for apple tissue, namely, a decreasing relaxation time for the main peak with increasing mealiness stage (from 458.6 to $104.4 \mathrm{~ms}$ ). The absolute value of the main $T_{2}$ peak derived from NMR relaxometry in whole apples is much higher than the average $T_{2}$ maps fitted from MRI images. This is expected because the MRI data is derived from a smaller number of echoes so that the main relaxation time peak fails to clearly resolve the contributions from the different subcellular water compartments (vacuole, cytoplasm and cell wall) [3]. The $T_{2}$ derived from MRI would therefore correspond to a weighed average of all three $T_{2}$ peaks.

\subsection{Characterization of Sample Mealiness with non-NMR Reference Measurements}

Table 4 shows a comparison of apple batches submitted to the accelerated storage protocol chosen to induce three potential degrees of mealiness and described above. The degree of mealiness was characterized by five parameters, namely, the Magness-Taylor tirmness, soluble-solid content ( $\left.{ }^{\circ} \mathrm{Brix}\right)$, shear crispiness, compression hardness and juiciness [5].

Table 4 shows that the range of variation (max-minimum in average batch values) in Magness-Taylor firmness and shear crispiness was higher in the 1999 batch (5.3 and $10.2 \mathrm{~N}$, respectively) than for the 2000 batch $(2.7$ and $4.8 \mathrm{~N}$, respectively). However, the range of variation in hardness was similar for both 1999 and 2000 batches ( $5.8 \mathrm{~N} / \mathrm{mm}$ in both cases). Surprisingly, both batches had a juicicess far above the conventional threshold for low juiciness (below $400 \mathrm{~cm}^{2}$ ). Indeed the 2000 batch was even more juicy than the 1999 batch. However, there is a slight increase in juiciness between mealiness level $0\left(567 \mathrm{~mm}^{2}\right)$ and mealiness level $1\left(668 \mathrm{~mm}^{2}\right)$ which tas also been reported in previous research studies [4]. The average values for the unknown batch lie between those of meaiy level 0 and $1\left(29.0 \pm 7.4 \mathrm{~N} / \mathrm{mm}\right.$ of hardiness, $30.3 \pm 7.6 \mathrm{~N}$ of crispness and $718 \pm 180 \mathrm{~mm}^{2}$ of juiciness) though there was a high variability between the fruit.

The high intra-batch variability encountered in the general 2000 experiment in comparison to 1999 - pointed to the presence of isolated mealy fruits. This was confirmed by computing the texture degradation score (0-11). A score exceeding 6 identifies the very mealy fruits and this identified 6 fruits out of 60 as being extremely mealy in the 2000 batch $(\mathrm{C} 0-1, \mathrm{Cl}-8, \mathrm{C} 2-12, \mathrm{C} 2-4, \mathrm{C} 2-5, \mathrm{C} 2-7)$. In the 
Table 4. Characterization of batches with reference measurements (averages with standard deviations).

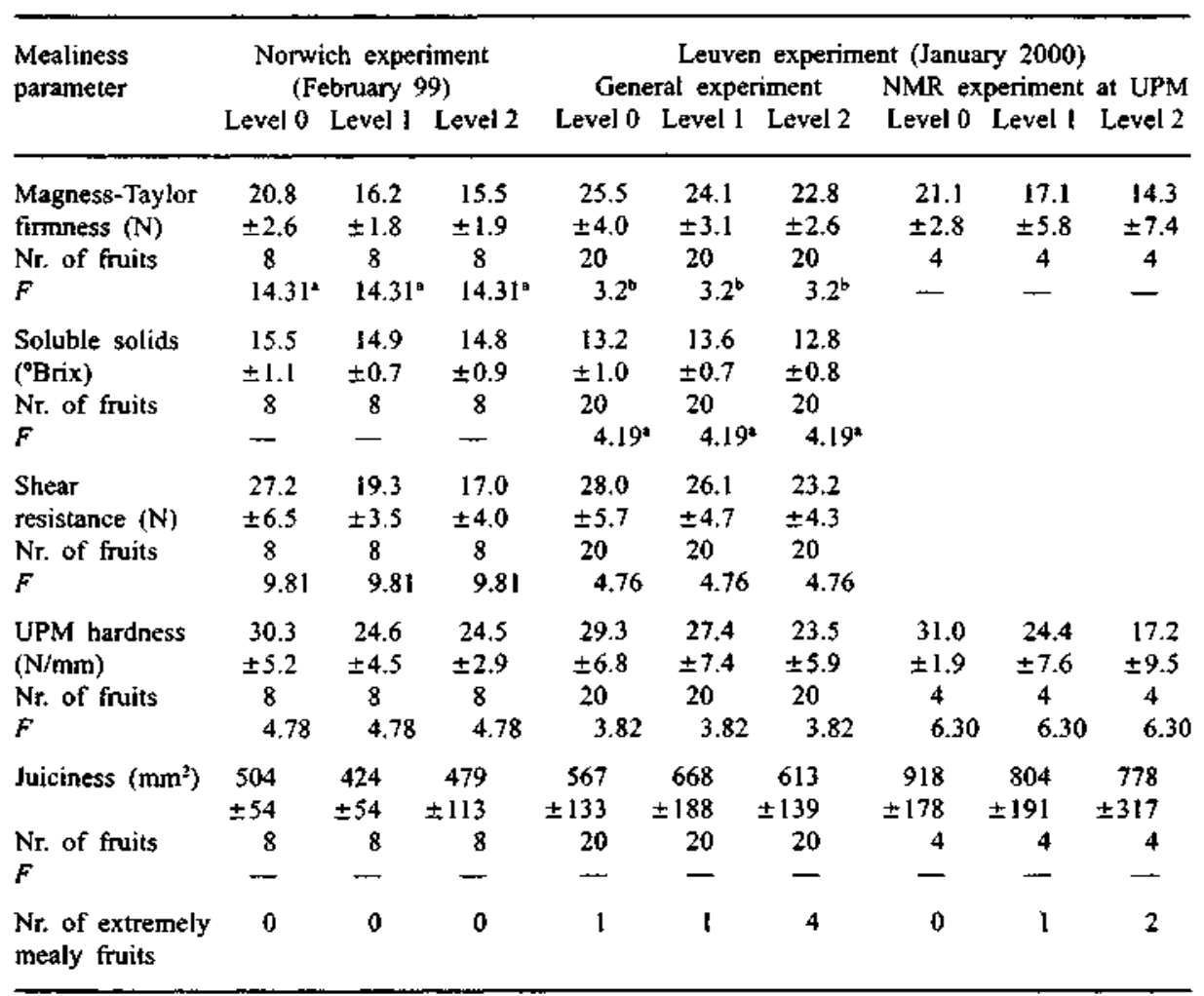

- The Fisher value for ANOVA value, $p<0.05$.

The Fisher value for ANOVA value, $p<0.01$.

unknown batch only 1 apple out of 20 (CUK-15) was very mealy by this criterion, whereas there were several extremely nonmealy samples in the 1999 batch. This erratic behavior on the onset of mealiness is one of the reasons why this physiological disorder has been traditionally difficult to address.

Table 4 also shows that the samples delivered to UPM showed a slight evolution, especially the samples of mealiness level 2 which achieved a batch average of $14.3 \mathrm{~N} / \mathrm{mm}$, near the bottom limit known for hardness [4]. The high intra-UPM batch variability also points to the presence of fruits undergoing different physiological stages (see the number of extremely mealy fruits in Table 4). Three out of twelve samples where identified as extremely mealy (C1-25, C2-26 and $\mathrm{C2}-27$ ). None of the samples from the unknown batch were identified as extremely mealy.

Table 2 shows that the range of variation of the reference measurements carried out in 2000 at UPM was much higher when calculated on a fruit-by-fruit basis than in the batch averages: $30.7 \mathrm{~N} / \mathrm{mm}$ compared to $13.8 \mathrm{~N} / \mathrm{mm}$ for hard- 
ness, and $764 \mathrm{~mm}^{2}$ compared to $140 \mathrm{~mm}^{2}$. The average of all the 16 standard errors per fruit for hardness and for juiciness are $31.9 \mathrm{~N} / \mathrm{mm}$ and $66 \mathrm{~mm}^{2}$, respectively (see Table 2).

A useful comparison of a non-NMR reference measurement to MRI or NMR relaxometry, which has shown to work on a batch basis, can also be done on a fruit-by-fruit basis provided the standard error of a measurement per fruit is not too different from that for batches. Because there were limited numbers of fruit samples and no repetitions this is unlikely to be the case in the experiments at Norwich and Leuven ( 8 fruits per batch in Norwich and 20 fruits per batch in Leuven, one replicate per fruit). However it is expected to be valid in the UPM experiment where there were 3 replicates per fruit. A metrological parameter defined as the ratio standard error over measured range $(S E / M R)$ was defined to illustrate this point and is listed in the last line of Table 2.

\subsection{Results: Multivariate Data Analysis}

Principal component analysis has been carried out on selected textural and MRI parameters for the NMR experiment at UPM (number of cases equals the number of fruits, 16, corresponding to the averages of three replicates per fruit). Figure 5 shows the projection of the variables on the first principal component plane (factor 1 vs. factor 2). Juiciness $\left(\mathrm{mm}^{2}\right)$ is seen to have a strong negative correlation with the standard deviation of $T_{2}$ maps as well as with the interquartile range of $T_{2}$ maps. These three parameters, which dominate the contribution to the first principal component, show no relationship with the second factor $\left(T_{2}\right.$ mode and lower quartile). Hardness $(\mathrm{N} / \mathrm{mm})$ and Magness-Taylor firmness $(N)$ are seen to be related to both principal components.

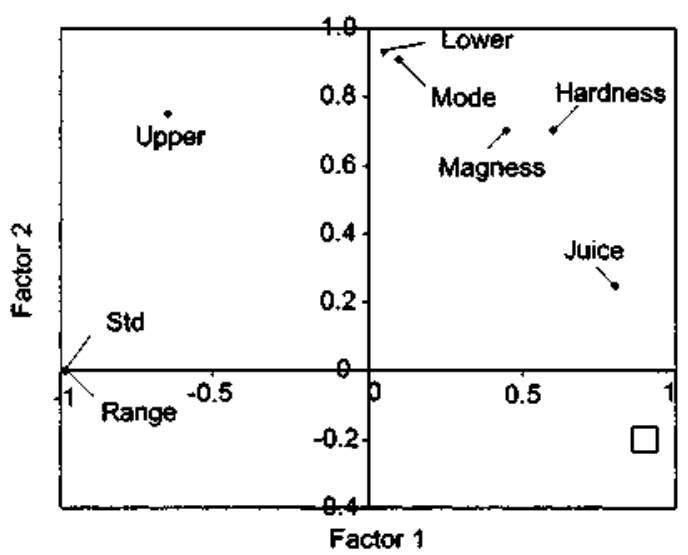

Fig. 5. PCA performed on textural and MRI parameters for the NMR experiment carried out at UPM. Explained variance at the first PCA plane: 0.84 . 


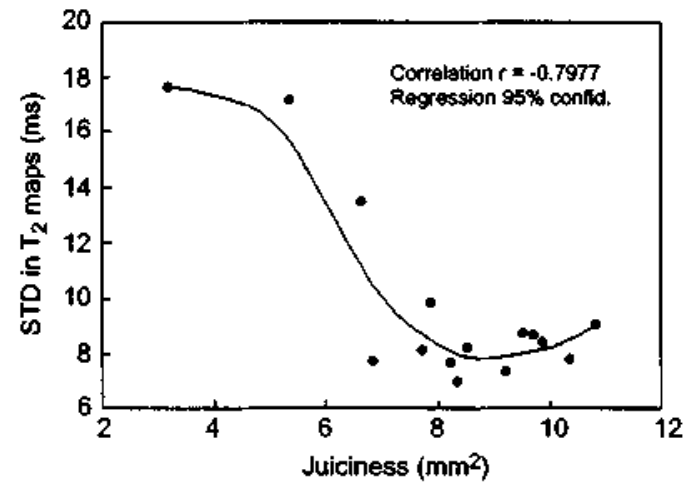

Fig. 6. Relationship between juiciness and standard deviation of $T_{2}$ maps. Each point is the average of three replicates.

Because mealiness results from a combination of tissue desegregation and dryness, one might expect correlations between the MRI relaxation behavior and both juiciness and tissue hardness and this is the case. A 2-D scatter plot of juiciness versus the standard deviation of $T_{2}$ maps reveals a nonlinear correlation (see Fig. 6); whereas a 2-D scatter plot of hardness (N/mm) versus the minimum value of the calculated $T_{2}$ histogram is linear (see Fig. 7).

The data from NMR relaxation obtained at UCM have not been included in the PCA because of the need to reject a whole work session resulting in a smaller data set ( 10 fruits with 3 replicates per fruit). Nevertheless there is a significant linear correlation between the main $T_{2}$ peak and the minimum, lower quartile and mode of $T_{2}$ maps ( $r=0.63, r=0.75$ and $r=0.79$, respectively), as well as with hardness $(r=0.82)$. These are much better results than those obtained when merging texture and NMR data in 1999, which can be traced to the low performance of the reference method in that season quantified as a high $S E / M R$ ratio (see Table 2).

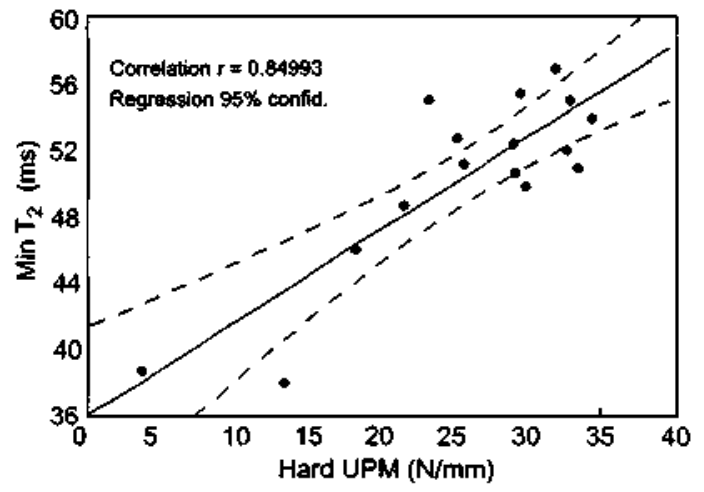

Fig. 7. Relationship between hardness and minimum value at $T_{2}$ maps. Each point is the average of three replicates. 


\section{Conclusions}

Our results have shown that the main water proton $T_{2}$ peak in apple decreases from 458.6 to $104.4 \mathrm{~ms}$ for whole apples ranging from 1 to 11 in texture degradation score (maximum range $0-11$ ) and that the $T_{2}$ measurement has a repeatability of $\pm 21.6 \mathrm{~ms}$. This is an encouraging result and suggests that there is a good prospect of using nondestructive, nonspatially resolved NMR relaxation measurements for rapid on-line mealiness assessment in apples.

Our analysis has also shown that a number of key features in the MRI $T_{2}$ maps of whole apples show significant correlations with the degree of mealiness. In particular, the histograms are non-Gaussian with extensive tails and the minimum $T_{2}$, lower quartile limit and $T_{2}$ mode are all smaller for mealy fruit than for fresh.

Our attempts to correlate different techniques such as textural measurements, MRI and NMR relaxation have highlighted a number of difficulties. In particular, we have shown that the ratio standard error over measured range calculated on a per fruit basis for each of the parameters needs to be below 0.1 before significant correlations can be made. When this criterion is fulfilled, we have found significant correlations between the NMR relaxation behaviour and both the juiciness and hardness of the fruit. These correlations are summarized in Figs. 6 and 7.

\section{Acknowledgements}

The EU is gratefully acknowledged (Project FAIR CT960302 and Concerted Action ASTEQ FAIRS CT97-3516) for financial support and J. Ruiz-Cabello for technical support on MRI.

\section{References}

1. Barreiro P., Ruiz-Cabello J., Fernandez-Valle M.E., Ortiz C., Ruiz-Altisent M.: Magn. Reson. Imaging 17, 275-281 (1999)

2. Barteiro P., Ortiz C., Ruiz-Altisent M., Ruiz-Cabello J., Fernández-Valle M.E., Recasens I., Asensio M.: Magn. Reson. Imaging 18, 1175-1181 (2000)

3. Hills B.P., Remigereau B.+ Int. J. Food Sci. Technol. 32, \$1-62 ([997)

4. Barreiro P., Ortiz C., Ruiz-Altisent M., De Smedt V., Schorte S., Bhanji Z., Wakeling I., Beyts P.K.: J. Texture Studies 29, 509-525 (1998)

5. Ruiz-Altisent M., Barreiro P.: Human Based Instrumental Measurement of Mealiness. Workshop on Mealiness in Fruits: Consumer Perception and Means for Detection. Katholike Universiteit Leuven, Belgium. 29th April 1999.

6. De Smedk $V_{\text {.: }}$ Ph.D. Thesis, Katholike Universiteit Leuven, Leuven, Belgium, 2000.

7. Ortiz C., Barreiro P., Correa E., Riqueime F., Ruiz-Altisent M.: Agric. Eng. 1, 1-40 (2000)

8. Provencher S.W., Dovi V.G.: J. Biochem. Biophys. Methods 1, 313-318 (1979)

9. Provencher S.W.: Comput. Phys. Commun. 27, 213-227 (1982)

Authors' address: Brian P. Hills, Institute of Food Research, Norwich Research Park, Colney, Notwich NR4 7UA, UK 